\title{
Massive MIMO with Nonlinear Amplification: Signal Characterization and Performance Evaluation
}

Abstract:

In this paper, we consider a $\mathrm{T} \backslash$ times $\mathrm{R}$ massive MIMO system with up to $\mathrm{R}$ simultaneous links ( $\mathrm{T} \gg>$ $\mathrm{R}>>1$ ) and nonlinear amplifiers in each transmitter branch. The impact of a nonlinear amplification is analytically studied and accurate expressions for the spectral characterization of the transmitted signals, as well as the bit-error-rate (BER) are derived. The accuracy of our analysis is validated by simulations. It is also shown that the nonlinear distortion levels for detection purposes decrease with the ratio $T / R$, which means that the impact of the nonlinear distortion effects on the system's performance can be alleviated provided that $T \gg>$. Therefore, the combination of massive MIMO systems with nonlinear, highly efficient power amplifiers can be an interesting option for low-cost, broadband wireless systems. 